Revue d'histoire de l'Amérique française

BRS REVUE D.HISTOIRE DE L'AMÉRIQUE FRANÇAISE

\title{
Le Père Pierre-Joseph-Marie Chaumonot, missionnaire de la Huronie (suite)
}

\section{André Surprenant}

Volume 7, numéro 3, décembre 1953

URI : https://id.erudit.org/iderudit/301608ar

DOI : https://doi.org/10.7202/301608ar

Aller au sommaire du numéro

Éditeur(s)

Institut d'histoire de l'Amérique française

ISSN

0035-2357 (imprimé)

1492-1383 (numérique)

Découvrir la revue

Citer cet article

Surprenant, A. (1953). Le Père Pierre-Joseph-Marie Chaumonot, missionnaire de la Huronie (suite). Revue d'histoire de l'Amérique française, 7(3), 392-412. https://doi.org/10.7202/301608ar d'utilisation que vous pouvez consulter en ligne.

https://apropos.erudit.org/fr/usagers/politique-dutilisation/ 


\title{
LE PERE PIERRE-JOSEPH-MARIE CHAUMONOT, MISSIONNAIRE DE LA HURONIE
}

\author{
$(\text { suite })^{*}$
}

\section{CHAPITRE III}

\section{DANS LE FEU DE L'ACTION}

"Mon apprentissage ainsi fait sous le père Daniel, écrit Chaumonot, je fus choisis par notre Père Supérieur (le père Jérôme Lalemant) pour accompagner a la Nation Neutre le père Jean de Brébeuf." Ils devaient être ainsi les premiers "à jeter les semences de l'Évangile dans la Nation Neutre"", après le Récollet, Joseph de la Roche Daillon, qui avait eu beaucoup à souffrir parce qu'il ne connaissait pas la langue. "Il estoit contraint d'instruire ceux qu'il pouuoit plustost par signes que de viue voix."

Depuis longtemps, les Supérieurs désiraient envoyer des missionnaires dans ces contrées; mais, instruits par l'expérience du Père Daillon, ils comprenaient qu'il fallait des ouvriers qui connus sent la langué.

"En outre, écrit le Père Lalemant, ce n'estoit pas l'ordre d'aller aux extremitez, sans passer par le milieu, et de s'appliquer à cultiuer les Nations plus esloignées, deuant que d'auoir trauailler aux plus proches. Ce qu'ayant esté fait les années precedentes nous nous

* Voir Revue d'Histoire de l'Amérique française, juin 1953: 64-87; septembre 1953: 241-258.

1. $\mathrm{AB}, 1: 53$.

2. ES, 3: 247 "Le P. brebeuf et le P. Chaumonot sont allez a la Nation Neutre nation ou on n'auoit point encor été porter l'Euangile," Charles Garnier à son frère, 23 juin 1641, JR, 20: 92-94.

3. Q, I (1641): 74 .

4. $Q, 1(1641): 71$. 
trouuasmes en estat, au commencement de l'Automne, de pouuoir destiner deux Ouuriers à cette Mission, sans faire aucun tort aux precedentes."

Chaumonot et Brébeuf partirent de la Maison de Sainte-Marie, le 2 novembre $1640^{\circ}$, accompagnés de deux domestiques français "tant pour les assister en leur voyage, que pour prendre le prétexte de trafiquer par leurs mains et passer comme marchands dans le pays en cas que sans cette considération les portes des cabanes leur deussent estre fermées, comme en effet il fust arriuet."

C'est à Saint-Joseph, dernier bourg des Hurons, qu'ils devaient s'approvisionner pour le voyage et rencontrer leurs guides. A cet endroit, ces derniers, en dépit de leurs promesses, ne se présentèrent pas. Fervente prière lancée vers le ciel. Vœu de Brébeuf. Aussitôt, il rencontre un jeune homme, qui n'avait aucun dessein de faire ce voyage; presque inconsciemment, Brébeuf l'aborde et lui dit: "Duio ack8e, sus allons-nous en de compagnie". Sans aucune résistance, il les suivit incontinent et il fut pour les missionnaires un très fidèle compagnon ${ }^{8}$.

Après quatre ou cinq journées de voyages, "c'est-à-dire enuiron quarante lieues, tirant tousiours droit au Sud", apparaissent les premiers bourgs de la Nation Neutre. Lalemant situe ainsi la région dite des Neutres:

Du premier bourg de la Nation Neutre, que l'on rencontre y arriuant d'icy, continuant de cheminer au Midy ou Sudest, il y a enuiron quatre iournées de chemin iusques a l'emboucheure de la Riviere si celebre de cette Nation, dans l'Ontario ou lac de S. Louys. Au deca de cette riuiere, et non au delà, comme le marque quelque Charte, sont la plus part des bourgs de la Nation Neutre. Il y en a trois ou quatre au delà, rangez d'Orient à l'Occident, vers la Nation du Chat ou Erioehronons.

5. Q, 1(1641): 71 .

6. $Q, 1(1641): 74$.

7. Q, 1(1641): 75 .

8. Q, 1(1641): 74-75.

9. Q, 1(1614): 71. “...Nation Neutre... qui est Eloignee de Notre Maison de cinq ou six Journées de Chemin dont il en faut coucher quatre dans la Campagne", Charles Garnier à son frère, 23 juin 1641, JR, 20: 92-94. 
Cette riviere ou Fleuue, est celuy par lequel se descharge notre grand lac des Hurons ou Mer douce, qui se rend premierement dans le lac d'Erié, ou de la nation du Chat, et iusques là elle entre dans les terres de la Nation Neutre, et prend le nom d'Onguiaahra, iusques à ce qu'elle se soit deschargée dans l'Ontario ou lac de Sainct Louys, d'où enfin sort le fleuue qui passe devant Quebek, dit de S. Laurens ${ }^{10}$.

On compte douze mille âmes dans toute l'étendue du pays, capable encore de fournir quatre mille guerriers, malgré les guerres, la famine et la maladie qui depuis trois ans ont régné en maître.

Ce sont les Français qui baptisèrent cette nation, nation Neutre, non sans raison, note le Père Lalemant, "car ce pays estant le passage ordinaire par la terre de quelque Nation d'Iroquois et des Hurons, ennemis iurez, ils se conservent en paix également avec les deux", tout en ayant cependant des guerres cruelles avec d'autres nations dites occi dentales ${ }^{11}$.

Ces Neutres, avec les Hurons et les Iroquois, viennent probablement d'une commune souche, mais par "succession de temps, ils se sont esloignez et separez les uns des autres, qui plus, qui moins, de demeure, d'interests et d'affections ${ }^{12}$."

Leur façon de "viure et de vestir" est semblable au mode d'être des Hurons. A la guerre, ils sont plus cruels que ceux-ci, car ils brulent les femmes prisonnières de guerre, comme ils le font pour les hommes. Ils pensent s'enjoliver en se maquillant le corps d'une manière pittoresque; "depuis la teste iusqu'aux pieds ils font faire mille diuerses figures auec du charbon picqué dans la chair, sur laquelle auparauant ils ont tracé leurs lignes: de sorte qu'on l'eur void quelquefois le visage et l'estomac figuré, comme le sont en France les morions et les cuirasses et les haussecols des gens de guerre, et le reste du corps à l'aduenant13". Quant au reste, notamment leur vie religieuse, politique et économique, ils ressemblent aux autres sauvages. Ils en diffèrent cependant par leur physique, car ils paraissent plus grands, plus forts et mieux faits.

10. $Q, 1(1641): 71$. Ragueneau situe aussi le pays des Neutres mais en rapport avec le pays des Hurons, $Q, 2(1648): 46$.

11. $Q, 1(1641): 72$.

12. $Q, 1(1641): 72$.

13. $Q, 1(1641): 73$. 
Ils ont, en plus un culte très poussé envers leurs morts, qu'ils entourent d'ure très grande affection. Les Hurons, après la mort des leurs, portent les corps au cimetière; ils les enlèveront de là pour la fête des Morts seulement. Les Neutres, eux, conservent leurs morts, jusqu'à ce que la pourriture des cadavres devienne réellement insupportable. C'est ainsi que les corps passent souvent tout un hiver dans les cabanes. Après, ils les font sécher sur un "eschaffaut" et ils retirent les os, qu'ils exposent, bien à la vue, dans leurs cabanes. Continuellement, ces "reliques" rappellent aux sauvages leur condition de mortels et attirent des cris lugubres et des lamentations rythmées, surtout de la part des femmes ${ }^{14}$.

Ils se distinguent encore par "la multitude et qualité des fols". En effet, quantité de sauvages se promènent par tout le pays, en imitant les extravagances des fous. Toutes les libertés leur sont permises, crainte de déplaire aux démons qui leur parlent en songe et qui leurs promettent grand succès à la pêche, s'ils obéissent. Ils causent, ainsi, beaucoup de dommages; par exemple, ils éparpillent la braise des foyers, ils brisent tout ce qu'ils rencontrent, comme des êtres furieux, quoique la plupart soient parfaitement conscients du rôle joué ${ }^{15}$.

Nos deux missionnaires, Brébeuf et Chaumonot, étaient partis, nous l'avons dit, le deux novembre. Ils couchèrent quatre nuits à la belle étoile, dans les bois, et, le cinquième jour, soit le 7 novembre, ils arrivèrent au premier bourg de la Nation Neutre, nommé Kandoucho, qu'ils nommèrent, eux, bourg de tous les Saints, en raison de l'octave de la Toussaint ${ }^{16}$. Ce voyage, comme tous ceux qu'entreprirent les missionnaires, avait été extrêmement pénible.

Voici le récit de Chaumonot:

Partant de notre Résidence au pays des Hurons, nous fîmes six jours de route, toujours dans le bois, et sans trouver aucun endroit pour nous reposer ou réfugier. Nous étions obligés de porter à dos tout ce qui nous était nécessaire pour notre nourriture. Les sentiers de ces forêts sont très-difficiles, etant fort peu battus, remplis de brousailles et de branches, coupés de marais, de ruisseaux, de rivières sans autres ponts que quelques arbres, brisés par l'âge ou par le vent. L'hiver

14. Q, 1(1641): 73 .

15. Ibid., 73.

16. Q, 1(1641): 75 . 
est la meilleure saison pour voyager, parce que la neige rend les sentiers plus unis. Mais il faut qu'elle soit durcie, comme nous l'avons trouvée à notre retour, à l'exception de deux journées: sans cela, on enfonce à chaque pas. Il y a encore un avantage à voyager en hiver: c'est que les cours d'eau sont glacés, et que nous avons pu, pendant soixante milles, trainer nos bagages. Il est vrai qu'on ne trouve aucun abri contre les vents qui sont très violents et très froids. Mais, grâce à Celui à qui la mer et les vents obéissent, nous avons pu marcher courageusement et joyeusement, malgré le froid, la fatigue et les chutes sans nombre sur la glace; ce dont mes genoux ont conservé bon souvenir. Mais qu'est-ce que cela en comparaison de ce que Notre-Seigneur a souffert pour moi? Je m'estimerais heureux de me briser bras et jambes à son service ${ }^{17}$.

Les deux missionnaires furent d'abord assez bien reçus, parce qu'ils passèrent en quelque sorte, auprès des sauvages, pour des prestidigitateurs. En effet, ils les convainquirent, par le moyen de l'écriture, qu'ils pouvaient connaître ce qui s'était fait ou dit dans des lieux éloignés. Voici leur expérience, telle que la raconte Chaumonot:

Le père de Brebeuf sortit de la cabane et alla assez loin. Cependant un de l'assembleo me dit d'un ton bas et en sa langue ses paroles: "Je vais à la chasse, je trouve un chevreuil, je prends une flèche dans mon carquois, je bande mon are, je tire et du premier coup j'abas ma proie; je le charge sur mes épaules, je l'apporte à la cabane, et j'en fait un festin à mes amis". Je n'eus pas plutôt ecrit ce petit discours qu'on rappela le père. On lui mit le papier en main et il lut mot pour mot ce qu'on m'avoit dicté. A cette lecture tous les assistants jettèrent un grand cri d'admiration. Ensuite ils prirent le papier et après l'avoir bien tourné et retournê, ils s'entre-disoient "Où est donc la figure qui représente le chasseur? Où le chevreuil est-il peint? Où est marqué la chaudière et cabane du festin? Nous ne voyons rien de tout cela, et pourtant l'ecrit dit à Héchon (C'est le nom sauvage qu'on avoit donné au père de Brébeuf et que j'ai eu l'honneur de porter après sa mort)". $\mathrm{Au}$ reste nous eûmes là une belle occasion de leur déclarer ainsi que nous fîmes que ce que l'écriture de nos ancêtres nous apprenoit de la foy étoit aussi véritable que ce que le papier écrit de ma main en leur présence avoit raconté à Héchon ${ }^{18}$.

17. Q, Carayon, 133-134.

18. $\mathrm{AB}, 1: 53-54$. 
Moyen simple et ingénieux, mais encore fallait-il y penser.

Avant de partir pour cette mission, Brébeuf avait écrit au Père Vitelleschi, Général de la Compagnie: "La moisson promet d'être abondante ${ }^{10}$." De fait, elle l'aurait été, si les calomnies odieuses des Hurons ${ }^{20}$ n'étaient venues briser le travail des deux missionnaires, qui se heurtaient à un mur de préjugés. Quand les Pères entraient dans les villages, ils trouvaient closes toutes les portes des cabanes. Le nom d'Echon retentit partout "comme celuy d'vn des plus fameux sorciers ou demons qu'on se fut iamais imagine". En l'absence du chef du pays, les capitaines refusèrent les présents des Pères; mais ils permirent aux missionnaires, en attendant son arrivée, de voyager librement dans le pays et de prêcher comme il leur plairait'21. Cependant, raconte Chaumonot, la défiance et les menaces les entouraient:

Pendant que nous étions paisiblement chez ces sauvages quelques uns des anciens Hurons qui nous attribuoient tout le mal que la petite vórole leur avoit causés envoyèrent deux députés aux Neutres, pour porter ceux-ci à se défaire de nous, parce que, disoient-ils, nous etions des sorciers et que nous prétendions ruiner la Nation Neutre en hyvernant chez elle, comme nous avions déjà ruiné la Huronne par nos sortilèges. Ils offrirent ensuite neuf haches pour récompense à ceux qui nous feroient mourir. C'étoit là un présent très considérable à ces peuples, qui étant assez oloignés des Hurons, où étoient les Français ne se servoient encore que de pierres pour couper du bois ou plutôt pour le rompre et le casser. Ces pierres étoient engagées dans la fente d'un bâton et liês avec une courroie à cette espèce de manche. Ainsi les neuf haches tentoient puissamment les Neutres, et o'est un miracle que nous ayons échappés à un si grand malheur.

19. Brébeuf à Vitelleschi, 20 aout 1640, Auguste Carayon, éd.: Première Mission des Jésuites au Canada, Lettres et documents inédits, 217.

20. $Q, 1(1641): 75-75$. Voici, entre autres, une de ces calomnies: “D'autres disoient que lorsqu'on auoit enterré cet excellent Chrestien Ioseph Chi8atenh8a, Echon se tournant du costé du pays des Sonnont8ehronons, qui l'avoient tué, dit tout haut: Sonont8ehronon, c'est fait de toy, tu es mort: et qu'aussi tost apres le Pere s'estoit acheminé vers leur quartier pour leur porter la maladie; laquelle en effet se trouuoit parmi les ennemis bien forte, pendant le seiour des Peres a la Nation Neutre. Surquoy les Hurons nous prioient de prendre bon courage et de faire mourir tous leurs ennemis, $Q, 1(1641): 76$.

21. Q. $1(1641) \cdot 75$. 
En effet un soir qu'on délibéroit de nos vies dans une assemblée de tous les notables du bourg, le père de Brébeuf faisant son examen de conscience eut cette vision. Un spectre furieux avoit dans ses mains trois dards ou trois javelots dont il nous ménaçoit tous deux qui étions ensemble en prières. Afin que l'effet suivit les menaces il lança contre nous un de ces traits, mais une main plus adroite, ou une vertu plus forte l'arrêta en chemin; et elle nous rendit le même bon office, lorsqu'il décocha le second et le troisième dard. Notre examen fini le père m'avertit du danger où nous étions et après qu'il m'eut raconté sa vision je jugeai comme luy qu'on pourroit bien tramer quelque chose contre nous. Sans en prendre l'alarme nous nous entro-confessames l'un l'autre et toutes nos prières achevées, nous nous couchâmes à l'ordinaire.

Bien avant dans la nuit, notre hôte revint du conseil, où les deux Hurons avoient présenté leurs haches pour nous faire casser la tête. A son arrivée dans sa cabane, il nous eveilla pour nous apprendre que par trois diverses fois nous avions été sur le point d'être massacrés, les jeunes gens s'étant offerts à faire le coup; mais qu'à toutes les trois fois les anciens les avoient tenus par la force de leurs raisons. Ce récit nous expliqua ce que le Père de Brébeuf n'avoit vu qu'en enigme ${ }^{22}$.

Brébeuf et Chaumonot eurent beau se rendre aux assemblées des sauvages, réfuter une à une toutes les calomnies lancées par les Hurons ${ }^{23}$, "le venin vne fois ietté, souligne Lalemant, ne sortoit pas si facilement du cœur de ces pauvres barbares qui craignent tout, pour ne pas connoistre celuy qui seul mérite d'estre craint et redoutét ${ }^{24}$ ". Au reste, les discours des Hurons avaient répandu, dans les esprits des Neutres, une frayeur incroyable. D'un naturel extrêmement défiant, surtout à l'égard des étrangers, ils l'étaient encore davantage envers des personnes, dont ils n'avaient entendu dire que du mal. Leurs oreilles et leurs cœurs étaient remplis des calomnies des Hurons. La seule vue des Pères, d'ailleurs vêtus d'une façon si différente de la leur, leurs démarches, leurs gestes et tout leur comportement extérieur semblaient confirmer ce qui avait été dit. Les bréviaires, l'écriture étaient, pour eux, autant d'instruments de magie et les prières des Pères, exercice de sorcier. Ils empoison-

22. $\mathrm{AB}, 1$ 54-56.

23. Voir Q, 1(1641): 76-77.

24. $Q, 1$ (1641): 76. 
naient les eaux du ruisseau, où ils lavaient leurs plats, disait-on; les enfants étaient saisis d'ure toux et d'un flux de sang, les femmes devenaient stériles, partout où les missionnaires passaient, etc..25 Par un exemple, Chaumonot confirme ce témoignage: "Je voulus une fois me mettre à genoux dans une cabane, où nous étions retirés pour prier avec plus de recueillement. Aussitôt, le bruit se répandit que Oronhiaguehre, c'est-à-dire, porte-ciel comme ils m'appellent, avait passé ure partie de la nuit à faire ses sortilèges et qu'en conséquence tous devaient se mettre en garde et se méfier de lui ${ }^{26}$."

La situation devint de plus en plus tendue et difficile, car personne ne voulait plus loger les missionnaires. La recherche d'un gíte, à l'abri du froid, provoqua une scène tragique, rapportée par Chaumonot:

Un soir que tout le monde étoit sur ses gardes et qu'on auoit comploté de ne nous point loger nous nous mîmes à la porte d'une cabane, à dessin de nous y glisser, lorsque quelqu'un en sortiroit. En effet une personne qui étoit dedans ayant ouvert la porte nous nous y fourrâmes aussitôt mais on ne nous eut pas plutôt apperçus qu'on pensa se pâmer de peur. Après qu'ils furent un peu revenus de leur crainte et qu'ils eurent repris leur esprit, ils envoyèrent avertir les anciens que nous étions chez eux et de la manière dont nous y étions entrés. Voilà incontinent la cabane pleine de monde qui vint au secours de nos nouveaux hôtes. - Les anciens nous entreprennent avec menace de nous mettre dans la chaudière, si nous ne délogions et si nous ne sortions de leur pays. Les jeunes gens pour appuyer les anciens disoient bien haut qu'ils étoient saouls de la chair noiratre de leur ennemies et que volontiers ils mangeroient de notre chair qui est plus blanche. Sur ces entrefaites un soldat armé d'arc et de flèches entre comme un furieux dans la cabane, bande son are et se met en devoir de décocher ses flèches sur nous. Je le regarde fixement et je me recommande avec confiance à St. Michel. Sans doute que ce grand Archange nous preserva puisque notre plus furieux ennemi s'apaisa presqu'aussitôt et qu'ensuite nos autres adversaires se rendirent aux raisons que nous leur donnâmes de notre arrivée et de notre séjour dans leur pays. Nous les as-

25. Q, 1(1641): 77-78. Voir aussi la lettre de Chaumonot à Nappi, 3 août 1641, Carayon, 134-135.

26. Carayon, 135. 
surâmes que notre unique prétention étoit qu'ils se soumissent à la foi que nous leur préchions pour les rendre saints dans le tems et heureux dans l'éternitéte.

Tout séjour plus prolongé étant maintenant intolérable, le Père Brébeuf jugea plus sage de déguerpir, de peur d'aigrir davantage ces sauvages.

Les deux missionnaires étaient demeurés quatre mois et demi; ils eurent le temps de visiter dix-huit bourgs, dont dix plus particulièrement; ils "proposèrent et publièrent" l'Evangile à trois mille personnes ${ }^{28}$. De nouveau nous pouvons leur appliquer ces ardentes paroles de saint Paul: "Tout bralant d'amour pour vous d'un amour semblable à celui de la mère qui entoure de soin ses petits enfants, nous étions prêts à vous donner, non pas l'évangile seulement, mais notre vie, tellement vous nous étiez devenus chers ${ }^{29}$." Restait à Brébeuf et Chaumonot la consolation d'avoir semé dans les larmes et les sueurs, condition essentielle pour une moisson abondante d'âmes, que d'autres, cependant, auront le plaisir de récolter.

$\mathrm{Au}$ retour, les deux missionnaires furent arrêtés au bourg de Teotongniaton, surnommé Saint-Guillaume, par une violente tempête de neige, qui les empêcha de continuer. Halte réservée par le ciel, pour procurer à ses serviteurs un grand réconfort. En effet, ils furent reçus par une hôtesse "qui s'estudioit de leur donner autant de contentement que tous les autres par le passé leur auoient donné suiet de desplaisirso". Elle poussa la délicatesse, jusqu'a leur préparer des plats spéciaux, parce qu'on était à l'époque du Carême. Elle instruisait même les Pères de la langue des Neutres, leur faisant écrire tous les mots, syllabe par syllabe, ainsi que de longues dictées, tout comme le maitre fait avec de petits écoliers ${ }^{31}$. "Ce fut sans doute vne prouidence de Dieu toute spéciale, écrit

27. $\mathrm{AB}, 1: 57-58$.

28. $Q, 1(1641): 78$. "Les petits enfants en danger de mort ont recueilli les premiers fruits de notre apostolat. Nous avons baptisé un grand nombre à l'insu de leurs, parents, qui s'y seraient opposés certainement." Chaumonot dans Carayon, 134.

29. 1. Thess. 11, 7-8. Phil., 1, 8.

30. Q, 1(1641): 78 .

31. $Q, 1(1641): 79$. 
Lalemant, que le retardement des Peres en ce lieu: car en vingt cinq jours qu'ils demeurerent en cette cabane, ils eurent le moyen d'aiuster le Dictionnaire et les Regles de la langue Huronne, à celle de ces Peuples et faire vn ouurage qui seul meritoit qu'on fist vn voyage de plusieurs années dans le pays, nos Sauuages se plaisans beaucoup plus auec ceux qui parlent leur propre langue, qu'auec ceux qui n'en font qu'approcher, qu'ils tiennent iusques là pour estrangers ${ }^{32}$."

Cette bonne sauvagesse subit alors une véritable persécution, tant des sauvages du bourg, que de ceux de sa propre cabane, qui la menaçaient de toutes les calamités possibles, sans cependant réussir à la dissuader. Ses petits enfants se battirent même avec leurs compagnons, pour la défense des Pères. Elle ne pouvait empêcher, à son grand déplaisir, les brimades infligées aux missionnaires. Un jour, par exemple, "vn fol de sa cabane se mit à cracher sur le Père Chaumonot, à lui déchirer sa soutane, à le vouloir brâler, à chanter tant d'iniures et à faire tant de tintamarres plusieurs nuits durant, que les Peres ne purent dormir ${ }^{33}$."

Enfin, les Pères réussirent à s'échapper de ce portique de l'enfer. Ils rentrèrent à Sainte-Marie, le jour de la fête de saint Joseph, à une heure assez hâtive pour pouvoir célébrer la messe, dont ils avaient été privés depuis leur départ ${ }^{34}$.

Après le martyre de Brébeuf, en 1649, Chaumonot racontera ce voyage du retour, qui ne le cède en rien aux autres; il trace les souffrances de Brébeuf, souffrances qu'il est à même de peindre pour les avoir ressenties et partagées:

32. Q, 1(1641): 79-80.

33. Q, 1(1641): 79 .

34. $Q, 1(1641)$ : 80 . Véritable mortification que cette privation prolongée de la messe. On s'en souvient, ils ne pouvaient même pas prier en remuant les lèvres. Mais, au milieu de ces souffrances, Brébeuf, le mystique, fut favorisé d'une vision racontée par Ragueneau: "L'année 1640, qu'il passa tout l'hiver en mission dans la nation neutre, une grande croix luy apparut quj venoit du costé des nations iroquoises. Il le dict au P. (is fuit Pater Josephus Chaumonot) quj l'accompagnoit: lequel luy demandant quelques particularités plus grandes de cette apparition, il ne luy respondit autre chose sinon que cette croix estoit sy grande qu'il y en avoit assez pour attacher non seulement une personne, mais tous tant que nous estions en ces pays, Arthur Melançon, éd., Memoires touchant la Mort et les Vertus des Pères I saac Jogues, Anne de Noue, Antoine Daniel, Jean de Brêbeuf, Gabriel Lalemant, Charles Garnier, Noel Chabanel \& un séculier René Goupil (Montréal, s.d.), 124. Désormais, l'abréviation sera: Mémoires. 
Le Pere Brebeuf jamais ne se plaignit, ny du froid, ny des incommodités qu'il souffrit, un hiver entier à la Nation Neutre... Je ne l'ay jamais veu dedaigner aucune sagamité pour mal assaisonnée qu'elle fust, froide, chaude, claire, \&... Estant à la Nation Neutre pour tous matelas, linceuls et couvertes pour se garantir des grands froids de la nuict, il n'avoit qu'une petite couverte, et avec cela exposé à tous les vents des cabanes sans endichon (lict d'escorce en forme de table sous laquelle les missionnaires couchaient).

A son retour de la Nation Neutre, il coucha 6 journées dans les bois, sur les neiges, sans aucun couvert de cabanes, pendant des froids qui faisoient craquer et fendre les arbres de la forest, avec un bruict semblable à des coups de mousquets, et avec tout cela, jamais ne fist semblant de souffrir froid $^{35}$.

Brébeuf, dit Chaumonot, ne se plaignait jamais ${ }^{36}$. Aussi, quand nous trouvons une phrase comme celle-ci, dans une lettre de Brébeuf: "Nous y avons beaucoup souffert: si quelques uns nous ont écouté volontiers, le plus grand nombre nous a repoussés, injuriés, menacés $^{37}$ ", il fallait que le degré de la souffrance humaine ait dépassé les limites de résistance naturelle. Aveu d'ailleurs corroboré par le Père Lalemant et Marie de l'Incarnation: "Bref, écrit le premier, il semble que les Pères fussent comme une balle de laquelle se iouoient les démons au milieu de cette barbarie, mais auec ordre de la diuine Prouidence, que rien ne leur manquast, comme en effect en quatre mois qu'ils ont été là, rien iamais ne leur a manqué de ce qui estoit

35. Mémoires, 142. "Pour comble des travaux de ce voyage, traversant durant deux journées un lac glacé, avec la charge des provisions qu'il falloit porter sur les espaules faute d'hotelleries, il tomba sur la glace d'une telle roideur, qu'il fut long temps sans se pouvoir remuer, ny relever. Enfin avec l'assistance d'un de ses compagnons, s'estant relevé, il se trouva sy affoibly du coup qu'il receut, qu'outre qu'il se rompit une des clavicules, dont il ne parla que deux ans après au chirurgien, il ne pouvoit plus souslever les pieds de terre, tant il avoit interdict l'usage des nerfs, en sorte qu'il fut contrainct, l'espace de 12 lieues de chemin quj luy restoient, de marcher ès lieux de plat pais, en traisnant ses pieds l'un après l'autre, sans les pouvoir lever de terre, et ès rencontres de montagnes, il marchoit à 4 pattes sur la neige, tandis qu'il montoit; et lorsqu'il descendoit, il s'asseoioit sur la neige, et puis de ses mains, il s'aidoit à se faire glisser jusques au bas des montagnes. Plusieurs foys ses compagnons de voyage le voulurent soulager, s'offrant à le traisner le reste du chemin, il s'en excusa tousjours. Mémoires, 143.

36. Mémoires, 142.

37. Brébeuf à Vitelleschi, 20 août 1641, Auguste Carayon, éd. Première Mission des Jesuites au Canada, Lettres et documents inédits, 217. 
nécessaire pour la vie, ny giste, ny nourriture suffisante, et se sont touiours bien portez parmy des peines et des incommoditez, qui se peuvent mieux conceuoir qu'expliquer"s8"; Marie de l'Incarnation rapporte de son côté: "Les RR. PP. de Brébeuf et Chaumonot ont jeté les premières semences de l'Évangile dans la Nation Neutre, où ils ont pâti jusqu'à mourir. Le R.P. Chaumonot a pensé avoir la tête fendue d'un coup de caillou...."39"

Déjà Chaumonot avait fait trois missions successives et très pénibles avec Ragueneau, Daniel et Brébeuf. Les souffrances s'accumulaient, mais sans lasser l'apôtre, toujours avide de sauver le plus d'âmes possible. Un véritable missionnaire ne se repose pas. Aussi, à son retour, en mars 1641, son supérieur le nomma "tantôt avec un Père, tantôt avec un autre". Il accompagne, une autre fois, le Père Antoine Daniel dans une visite à la mission Saint-Joseph. Un jour, au bourg Saint-Michel, il baptisa une jeune femme mourante. Cet acte, qui ouvrit les portes du ciel à une Huronne, faillit lui coûter la vie. Avec son humilité coutumière et son esprit surnaturel, Chaumonot raconte cette tentative d'assassinat:

Un des parents de la malade, irrité contre nous a cause de ce baptême nous attend dehors à l'entrée de la cabane avec une grosse pierre en main pour nous la décharger de toute sa force sur la tête, lorsque nous sortions. Par bonheur pour moi je passe le premier et voila qu'au moment que je mets le pied dehors ce furieux m'abattit mon chapeau d'une main et de l'autre il me frappe de sa pierre sur la tête nue: je fus tout étourdi du coup, et l'assassin voulant m'achever prit une hache. Mais le Père Daniel qui étoit fort adroit, la lui arracha des mains. On me mena chez notre hôte, où un autre sauvage fut mon charitable médecin. Ayant vu la grosse tumeur que j'avois à la tête, il prit une autre pierre pointue pour m'y faire des incisions par lesquelles il tacha d'exprimer tout le sang meurtri et puis il arrosa le haut de la tête aveo de l'eau froide, dans laquelle il avoit quelques racines pilées. Il prenoit dans sa bouche cette liqueur médecinale et la souffioit dans les plaies ou dans les ouvertures qu'il avoit faites. Cette cure fut si heureuse qu'en fort peu de tems je fus guéri. Dieu se conten-

38. Q, 1(1641): 78 .

39. ES, 3: 247. Marie de l'Incarnation est la seule à rapporter ce dernier fait; peut-être mentionne-t-elle la tentative d'assassinat sur Chaumonot, lors de son aéjour au bourg de Saint-Michel avec Antoine Daniel. Voir la note 40 de ce chapitre. 
ta du désir que j'avois du martyr ou plutôt il ne me jugea pas digne qu'on me fit mourir en haine du premier de nos sacrements ${ }^{40}$.

Dieu, en effet, conservait son serviteur pour un apostolat fructueux auprès des âmes. Chaumonot serait privé de la gloire du martyre sanglant, mais il allait acquérir celle du martyre quotidien d'une longue vie de cinquante-deux années, toute remplie d'abnégation et de sacrifices.

Chaumonot et Daniel continuèrent leur mission errante, visitant, l'un après l'autre, les différents bourgs. Chez les "Arendaenhronons"'11, ils séjournèrent, cependant, plus longuement. Les Pères espéraient une conversion plus rapide de ces sauvages parce qu'ils ne rendaient aucun culte à quelque fausse divinité, "puisque, affirme Lalemant, comme sur vne table rase, n'ayant rien à effacer, on y pourroit sans résistance imprimer les idées d'vn vray Dieu, et les conduire au respect et à l'adoration qui luy est deue par toute la Terre"12."

Les missionnaires furent rapidement déçus. Car l'expérience leur fit bientôt voir que ces sauvages étaient aussi imbus de superstitions diaboliques. Tout le bonheur de leur vie dépendait de leur songe qu'ils prenaient pour des divinités. En outre, ils reconnaissaient des génies puissants, qui disposaient des affaires publiques, causaient les famines, provoquaient les guerres et accordaient la victoire aux sujets plus fidèles. Souvent même, ces démons se rendaient visibles, de façon certaine.

Le Père Lalemant prouve, ensuite, que ces sauvages sont réellement devenus esclaves des démons. Voici, parmi plusieurs, un cas:

Cet Hyver, vn ieune homme enuiron de trente ans, vit entrer sur le soir vn spectre en sa Cabane, en forme de Megere, armee de tisons et de flammes, qui s'escrioit qu'on le bruslast. Son esprit est trouble aux horribles regards de cette furie onragee; il entre luy-meme en fureur, se iette dans les foux qui estoient allumez; et quoy qu'il se bruslast, il n'en ressent point la douleur. Il chante continuellement plusieurs iours, ou

40. $A B, 1: 59-60$. Le même fait est raconté dans $Q, 1(1641): 81$.

41. La première des quatre nations huronnes découvertes par les Français. Les "Arendaenhronons" gardent un souvenir excellent des bons exemples de Champlain, surtout de sa chasteté. Tous les Français ne furent pas aussi édifiants et plusieurs nuisirent énormément au travail des missionnaires. Voir Q, 1(1640): 90 .

42. $Q, 2(1642)$ : $82-83$. 
plustost il pousse sans cesse des hurlements terribles, sans toutefois perdre rien de sa voix. En fin on saisit cet homme forcené, on interroge ce Demon de fureur qui l'anime et qui le possede. Il répond qu'il demande en offrande vne armure sauuage, qui le couure de pied en teste, vne enseigne de muzeau de loup, et quelque autre equipage de guerre ${ }^{43}$.

A force de zèle et de prières, les Pères réussirent à ravir quelques ames aux démons. Parfois, ces nouveaux chrétiens leur donnent par leur vie pure, en tout exemplaire, de bien douces consolations. Ainsi le sauvage Jean-Baptiste, premier adulte en pleine santé, baptisé a la mission Saint-Jean-Baptiste, qui disait à ses compagnons:

Vous sçaurez maitenant que ie suis baptise, et que ie ne rougiray iamais d'estre appelle Chrestien. I'admire vos courages, d'estre sans crainte et de ne point trembler de peur, aux nouvelles qu'on nous apporte de ces feux Eternels, qui bruslent à iamais au centre de la Terre ceux qui durant leur vie n'ont pas adoré cet Esprit Tout-Puissant, qui d'vn mot a cot la Terre et les Cieux. Pour moy ie confesse ma laschete: ces nouvelles m'ont d'abord fait trembler, et l'horreur qui me saisit lors que i'y pense, m'a fait à juste raison prendre la résolution d'euiter ce malheur, maintenant qu'il est en mon pouvoir...

Non, non, mes Freres, ie n'ay plus de oourage, quand on viendra m'inuiter au peché, ie trembleray de peur et d'appohension, en cela ie vous laisseray tout seuls dans le peril; mais s'il faut attaquer l'Ennemy, s'il faut venger la mort de noe parens, s'il faut defendre le Pais, ie vous tiendray fidele compagnie, ie ne fuyray pas le danger, et i'espere que le courage ne me manquera pas à l'oocasion ${ }^{44}$.

En vain, ses amis essayèrent de l'engager dans les superstitions du pays. Son fils étant tombé malade, il refusa tous les remèdes diaboliques des sorciers. Sa femme l'abandonna, amena avec elle l'enfant et prit un autre mari. Malgré tous les assauts, sa chasteté fut alors exemplaire: fait d'autant plus méritoire, qu'en ce pays, la pudeur des femmes passe pour un opprobre et l'honneur d'une vie vertueuse, un déshonneur; d'autant plus méritoire encore, qu'il avait vécu ce genre de vie depuis sa jeunesse. La crainte de Dieu fut sa défense. "Il a tremblé de peurts."

43. $Q, 2(1642): 83$.

44. $Q, 2(1642): 85$.

45. Q, 2(1642): 85. 
Au bourg Saint-Michel, également visité par Chaumonot, la situation était identique et offrait deux tableaux contrastants: des âmes sous le joug de Satan, incapables de sortir de cette captivité et quelques baptisés, délivrés de l'esclavage, qui sont visiblement protégés par le $\mathrm{Ciel}^{46}$. Les missionnaires avaient sous les yeux la vision des deux étendards, que saint Ignace présente à ses retraitants dans les Exercices Spirituels.

Les missionnaires, nous l'avons vu, parcouraient plusieurs bourgs dans ces missions errantes. Pendant les années 1643-1644, Chaumonot s'occupa de la mission de "Saint-Michel aux Tahontaennat 47" avec le Père François du Péron ${ }^{48}$. Le Père Jérôme Lalemant, supérieur des missions huronnes passa lui-même deux mois de l'hiver avec eux ${ }^{40}$.

Là encore, les Robes noires furent victimes des calomnies.

46. $Q, 2(1642)$ : 87-88.

47. Il semble qu'il donna tous ses soins a cette mission car les Relations ne le mentionnent nulle part ailleurs. Le P. Lalemant écrit:... Il y fallut dresger une chapelle et y establir une Mission plus a demeure que nous n'auions fait iusques alors. Le P.J.M. Chaumonot et le P. François du Peron en ont le soin, et Dieu m'a donné la consolation enuiron deux mois de l'hyver d'y voir les premieres ferueurs de cette Eglise, Q, 2(1641): 94.

48. Né à Lyon, le 26 janvier 1610 , il se joignit à la Compagnie de Jésus à Avignon le 23 février 1627 . Il arriva a Québec le 30 juin 1638 et mourut le 10 novembre 1665 a Chambly. Ses restes ont été transportés dans la chapelle du collège de Québec, le 16 novembre 1665 , Melançon, 29. Au sujet de la découverte de ses ossements faite à Québec, voir Rochemonteix, 1: 456-465. Voir aussi le récit de sa mort dans C.-H. Laverdière et H.-R. Casgrain, éd., Le Journal des Jesuites (Québec, 1871), 338.

49. Il fut alors un témoin plus immédiat des labeurs de ses missionnaires, de sorte qu'il pouvait leur rendre ce splendide témoignage dans une lettre au Provincial de France, le P. Binet:... "et s'il y a eu quelque bien dans les commencemens de la conuersion de ces Peuples, il faut aduouer qu'apres Dieu tout est deu aux travaux de nos Peres, dont Notre Seigneur a voulu que j'aye esté tesmoin, voyant la ferueur de leur zele, leur courage indomptable, leur patience a tout souffrir, leur actiuité a tout faire, leur humilité dans vne vie vrayement cachée en vn monde inconnu, personnes qui d'ailleurs ne manquent pas pour la pluspart de qualitez qui les eussent rendus recommandables en France. Quand ie les voy embrasser la Croix auec plaisir, les souffrances auec ioye et les mépris auec amour, qu'ils portent chaque iour leur ame entre leurs mains, estant continuellement exposez a mille dangers de la mort, et que peut-estre la pluspart sont pour mourir au milieu des feux et des flammes d'vn ennemy cruel, qui va de iour en iour rauageant ces Pays; quand ie voy que ces dangers les animent plustost que d'affoiblir le moins du monde leur courage, il me vient souuent en pensée que Dieu vouloit qu'vne vertu si forte, si constante et si vigoureuse, suppleast au defaut des miracles, dont il me semble que sa diuine Prouidence ne veuille pas se seruir en ces siecles derniers, pour aduancer la conuersion de ces terres infidelles, Q, 2(1645): 51 . 
En cet endroit, c'est un Algonquin, qui, ayant hiverné avec les Hurons, jeta la terreur parmi les sauvages, par tous ses discours mensongers:

Il esbranla tellement les esprits, raconte Lalemant, et leur donna des craintes si puissantes de ces malheurs dont il les menacoit, que la terreur en fut incontinent respandue dans le bourg. Les impies triomphèrent alors, les foibles perdirent courage, et plusieurs qui sembloient n'estre pas esloignez du Royaume de Dieu prirent le dessein d'attendre et de voir quel succez auroit la Foy dans les autres qui y demeuroient engagez. Les Chrestiens cependant tiennent bon, leur courage s'anime, ils parlent aussi haut que iamais, et nous voyons en cette Eglise que si le Diable a du pounoir sur ceux qui ne sont pas sortis encore de sa captiuite par le sacrement du Baptesme, ces eaux sacrées esleuent vne âme au dessus des craintes terrestres, et font qu'elle ne redoute que Dieu et le pech $\theta^{50}$.

Remarquons ce fait: Les Pères changent de bourgs, mais les dispositions et les réactions des sauvages ne se modifient pas. Cependant, Chaumonot demeure enthousiaste, comme le prouve cette lettre écrite à Marie de l'Incarnation et citée par elle-même à son fils: "Si ces deux hivers prochains les conversions continuent comme ces deux précédents, nous espérons que les chrétiens deviendront les plus forts en ces cinq bourgs, et en peu de temps, ils attireront après soi non seulement leurs concitoyens, mais encore tout le reste du pays et voire tout le pays des Hurons ${ }^{51}$."

Par la suite, nous perdons les traces de Chaumonot jusqu'en 1647, où il apparaît à Saint-Ignace, sous le nom huron d'Aronhiatiri, qui signifie porte-ciel ${ }^{52}$. De plus, dans l'autobiographie, il y a un vide de sept années, soit de 1641 à $1648^{53}$. Sachant que les Pères Du Péron et Chaumonot furent missionnaires au même endroit, comme nous l'avons vu plus haut, nous pourrions expliquer ce silence par deux hypothèses: ou bien le Père Du Péron est demeuré

50. Q, 2(1644): 96.

51. Chaumonot à Marie de l'Incarnation dans ES, 3: 382.

52. Nous avons vu que les Neutres avaient surnommé Chaumonot Oronhiaguehre, c'est-à-dire porte-ciel. Voir le long développement très scientifique dú Père Jones, prouvant que ces deux noms, huron et neutre, désignent vraisemblablement le Père Chaumonot. Jones, 371-374.

53. Voir chapitre premier, note 51. 
a Saint-Michel jusqu'en 1646; ou bien le Père Chaumonot fut missionnaire résidè.t jusqu'en avril $1648^{\text {H4 }}$.

Toutefois, les auteurs des Relations nous permettent de connaitre le climat gér éral dars lequel a vécu Chaumonot, pendant ces années 1644-1645-1646. Avant de céder son paste de Supérieur au Père Raguereau, le Père Lalemant trace, le 15 mai 1645, un tableau général peu séduisant:

Les maladies se sont suiuies les vnes apres les autres, et il sembloit qu'elles en voulussent plus aux Chrestiens qu'aux Infidelles, dépeuplant plus cruellement leurs familles, et pardonnant le plus souuent à ceux qui auoient refusé le Baptesme, en mesme temps que dans vne mesme cabane et dans un mesme lict, la mort nous ravissoit les autres qui auoient embrassé la Foy.

Les famines ont ou leur tour; ot on a orế qu'ayant change de Maistre et que mettant ses confiances plus tost en Dieu qu'aux Demons de l'Enfer, la Foy auoit attiré ces malheurs apres soy, et que celuy qu'elle adoroit, estoit ou impuissant a nous faire du bien, ou qu'il manquoit d'amour pour coux qui vouloient on auoir pour luy.

Les guerres ont esté plus impitoyables, et quoy qu'elles ayent esté rauageant dans lour fureur plus cruellement $\infty$ Pays, sans pardonner a aucun sexe, à aucun age n'y à aucune condition de personnes, toutefois nous pouuons dire en vérité, qu'il semble que Dieu ait voulu moissonner la fleur de nos Eglises par oo glaive tranchant ${ }^{55}$.

Puls, sur une note plus optimiste, il continue:

Car nonobstant tous ces ravages de pestes, de famines et de guerres, quelque opposition qu'ayent ces peuples en leur naturel, en leurs loix et en leurs coustumes à la sainoteté do la Foy, quelque Empire qu'y ayent les Demons, nous n'avons pas laissé chaque année d'en baptiser bon nombre, et encore oette dernière annóe plus de cent septante... Ce qui nous fait dire, sans qu'il nous en reste aucun doute, Digitus Dei est hic. Or si Dieu est pour nous, pourrions nous oraindre au milieu

54. Jones, 350-351. En 1645, cependant, selon la Relation de 1646, il n'y eut aucun missionnaire a Saint-Michel pendant un long temps. "Dieu permit que le Pere qui auoit soin de cette Mission, fut obligé de s'en absenter bien long-temps, eans que nous puissions y suppléer par vne autre voye, plusieurs de nos Peres estans tombez en mesme temps malades, et les autres necessaires autre part." $Q, 2(1646)$ : $77-78$.

55. Q, 2(1645): 39 . 
de nos entreprises, sans nous exposer aux reproohes que fit lo Sauueur du monde à S. Pierre: Modicae fidei, quare dubitasti ${ }^{\text {s6 }}$.

L'année suivante, 1645-1646, au dire de Ragueneau, fut plus heureuse: "La terre leur a esté plus liberale que l'an passé, le bled d'Inde qui est le principal de leurs richesses, estant venu quasi pour tout à ure heureuse maturité. Les lacs et les riuieres leur ont fourny du poisson auec abondance. Le trafic qu'ils ont eu auec les nations éloigrées, ne leur a pas mal reussy". Et "les maladies contagieuses qui alloient dépeuplant nos bourgades, les laissent maintenant en repos". Il n'y a que "la guerre qui tient les affaires en balance: car elle cortinue tousiours auec les quatre nations Iroquoises plus voisires de ros Hurors... Ie veux dire que dans les diuers rencontres que nos Hurors ont eus depuis vn an auec leurs ennemys, les succez de leurs armes ort esté partagezs"'. Pour tout résumer, encore avec Raguereau: "L'Eglise naissante est comme une armée, elle se fortifie elle-même avec gloire plus elle est attaquéess."

Il faut noter aussi que, dès son arrivée, le Père Ragueneau transforma en résidences, les missions données aux bourgs de la Conception, de Saint-Joseph, de Saint-Ignace, de Saint-Michel, de Saint-Jean-Baptistess. A chacun de ces endroits, des chapelles furent construites. Avant même le son de la cloche, qu'on avait suspendue, les sauvages se réunissaient pour la messe et les prières du soir. Tous se confessaient chaque semaine et ils se préparaient, deux ou trois jours d'avance, à passer saintement le dimanche et, plus spécialement, ceux qui devaient s'approcher de la Sainte Table. Le dimanche midi, ils s'assemblaient de nouveau pour le sermon ou le catéchisme, qui était suivi de la récitation du chapelet. L'assiduité et la ferveur des sauvages de ces missions étaient remarquables. Le Père Ragueneau déclare que, cette année-là, les missionnaires baptisèrent cent soixante-quatre personnes ${ }^{60}$.

56. Q, 2(1645): 43. Le Père Lalemant poursuit en racontant les exploits des baptisés. Voir tout ce chapitre, $Q, 2(1645)$ : 38-52.

57. Q, 2(1646): 54 . On sait que les années des Relations commencent en mai, juin ou juillet.

58. Q, 2(1646): 56 .

59. Ibid., 56.

60. Q, 2(1646): 56-57. 
Maintenant admis que le surnom "Aronhiatiri" désigne Chaumonot, nous le retrouvons en 1647, à Saint Ignace I avec le Père René Ménard ${ }^{61}$. Ce nouveau compagnon de Chaumonot était tenu en très haute estime par ses confrères. On ne tarit pas d'éloge à son sujet: "Religieux très fervent de notre Compagnie ${ }^{62}$ ". "L'idéal du dévouement surnaturel ${ }^{63 "}$ " "Véritablement, la Nouvelle-France n'avait point alors de missionnaire plus accompli. Le ciel l'avait particulièrement doué d'un talent rare pour s'insinuer dans l'esprit des sauvages". "Il passait ses nuits à prier et ses jours à se dévouer; il avait coutume de dire: "Mes Pères nous n'en faisons que trop, mais nous n'en faisons pas assez pour l'amour de Dieu ${ }^{64}$."

Pendant un certain temps, Chaumonot et Ménard travaillèrent ensemble; puis, Chaumonot demeura seul et fut remplacé ensuite par le Père de Brébeuf ${ }^{65}$. Nous ne savons rien du travail particulier de ces apôtres, bien que nous connaissions les vertus héroiques des néophytes de Saint-Ignace I, qui parlent par elle-mêmes de l'heureuse influence des missionnaires ${ }^{66}$. C'est une autre lettre du Père Lalemant, pour lors Supérieur à Québec, qui nous fournit un aperçu de la situation:

La Relation de cette année que i'enuoye à vostre Reuerence, seruira de confirmation, que l'estat de la vie presente est le regne de l'instabilité, du trouble et de l'obscurité, et que tous les temps et les lieux sont remplis des iugements de Dieu incompréhensibles à nos esprits, et que les routes et les chemins de sa Diuine Majesté pour arriuer à un but sont bien différens de ceux que les hommes auroient choisis. Ces deux dernières

61. Parisien, né en 1605, le 7 septembre. Entré chez les Jésuites, le 7 novembre 1624 , il arriva z̀ Québec le 8 juillet 1640. Il mourut de faim, le 7 ou 8 aout 1661, dans les bois du Wisconsin, lors d'une mission chez les Outaouais, ES, 3: 165, n. 4; Melançon, 57. Voir sa lettre d'adieu, très touchante, adressée à un ami et datée de TroisRivières, le 27 août 1660 , à deux heures après minuit dans $Q, 3(1660): 30$. Voir aussi le récit tragique de sa mort dans $Q, 3(1663): 17-25$ et $R A, 240-243$. Résumé de sa vie dans T.J. Campbell, Pioneer Priests of North America, 1:159-172.

62. $Q, 3(1657)$ : 54 (Ragueneau).

63. A. Gosselin, Vie de Mgr. De Laval, premier évêque de Quêbec et apôtre du Canada, 1622-1708 (2 vol., Québec, 1890), 1: 274.

64. Rochemonteix, 1: 429.

65. Jones, 359; 367. Le Père Ménard était à Saint-Ignace 1, au moins depuis 1646, car le Père Garnier, dans une lettre du 14 mai 1646 dit: "Le P. Ménard est maintenant au bourg de Saint-Ignace", Jones, 359-360.

66. Voir Martin, 86-90, qui résume $Q, 2(1648)$ : 65-68. 
annees, les fleurs de la paix auec les Hiroquois, nos ennemis, nous en auoient fait esperer des fruits agreables et une heureuse récolte; mais la perfidie de ces barbares suruenant làdessus, comme une gresle sur un champ prest à moisonner, semble auoir vn peu retardé et reculé nos espérances ${ }^{67}$.

Il est certain, toutefois, que le zèle de nos missionnaires est pour une bonne part, dans cette déclaration de Ragueneau: "la mission de Saint-Ignace, plus nouuelle que les precedentes, est dans vne ferueur et vne innocence qui estonne les Infideles, et que iamais nous n'eussions pensé voir en si peu de temps dans les commencemens d'vne Église ${ }^{68}$."

Derechef, les espoirs des missionnaires devaient être trompés. A la fin de l'hiver de 1648, entre le mois de février et le 16 avril $1648^{69}$, quelque trois cents habitants du bourg de Saint-Ignace, hommes et femmes, s'étaient retirés dans les bois, pour la chasse. Une troupe de "Sonnontoueronnons" tua sept personnes et amena vingt-quatre prisonniers. Près de trois cents sauvages retournèrent sur les lieux et pour enterrer leurs morts et pour rapporter le fruit de leur chasse. S'étant divisés, çà et là, ils furent surpris par les Iroquois "Annieronnons"; environ quarante personnes furent tuées ou emmenés captives. Ces sauvages hurons, craignant d'être entièrement anéantis, démolirent leur petite chapelle de Saint-Ignace I et ils allèrent établir, près de la résidence centrale, un autre bourg, nommé Saint-Ignace $\mathrm{II}^{70}$. Ce départ précipité du bourg de SaintIgnace I donna lieu à une scène pathétique très impressionnante, narrée par Ragueneau: Ignace Onakonchiarink, chez lequel demeuroit le missionnaire de ce bourg ${ }^{71}$,

ayant remarqué quelque tristesse sur le visage du Pere, s'en alla devant l'Autel, où apres auoir demeuré en prieres vn

67. $Q, 2(1647): 1$.

68. $Q, 2(1648): 60$.

69. Jones, 367.

70. $Q, 2(1648): 49-51: 65$. "Mais quand le village voisin, nommé Saint-Jean Baptiste, qui était sur la frontière à l'est, eut jugé prudent de se transporter ailleurs, Saint-Ignace se trouva découvert et exposé à la première invasion de l'ennemi. Dans cet isolement, la résistance était bien difficile, malgré la bravoure de ses guerriers, malgré le fossé naturel qui les protégeait de trois côtés, et malgré son enceinte de pieux élevés. Les capitaines résolurent de changer le village de place, et de le transporter plus près du poste français de Sainte-Marie", Martin, 86.

71. Chaumonot était donc seul missionnaire à ce moment-là. 
temps notable, il s'approoha du Pere et luy tint ce discours, auquel ie ferois conscience d'adiouster aucun mot. Aronhiatiri, lui dit-il, (o'est le nom que les Hurons donnent au Pere), i'ay l'esprit tout abattu, non pas de mon affliction, mais de la tienne. Tu t'oublies ce semble de la parole de Dieu que tu nous presches tous les iours. Ie me figure que la tristesse qui paroist sur ton visage vient de nos afflictions, ce que cette Eglise qui estoit si florissante va se dissiper: on va abattre cette chapelle: plusieurs de no frères Chrestiens sont morts ou captifs; ceux qui restent vont se disperser de tous costez, en danger de perdre la Foy. N'est-ce pas là ce qui te trouble? Hélas mon frere, adiousta-t-il, est-ce à nous à vouloir sonder les desseins de Dieu et pouuvons-nous bien les comprendre? Qui sommes-nous? vn rien. Il sçait bien ce qu'il faut et void plus clair que nous. Sçais-tu ce qu'il fera? Ces Chrestiens qui se vont dissiper porteront leur Foy auec eux et leur exemple fera d'autres Chrestiens où il n'y en a point encore. Pensons seulement que nous ne sommes rien, que nous ne voyons goutte et que luy seul sçait nostre bien. C'est assez, ie t'asseure, pour me consoler en mon aduersité, me voyant miserable de tout point, de penser que Dieu aduise a tout, qu'il nous aime ot scait bien ce qu'il nous faut. Il poursuiuit dans cet air un demy quart d'heure, et le Pere admirant vne Foy si entiere dans le coeur de co bon Sauuage, et cet esprit vrayment Chrestien, en benit Dieu; et n'ayant point d'autre pensée, sinon que Nostre Seigneur luy auoit mis ces paroles on la bouche pour sa consolation, il ne pat se tenir les larmes aux yeux de l'embrasser, et luy dire qu'en effet il le consoloit solidement, que ce qu'il disoit estoit veritable et qu'il parloit on la facon que les Chrestiens se doiuent consoler dans lours afflictions... ${ }^{72}$

Scène touchante, qui traduit bien le caractère, simple et sensible, de Chaumonot. Ces premiers malheurs, arrivés a Saint-Ignace I, marquaient seulement le début de la destruction systématique de la Huronie, entreprise par les Iroquois. Chaumorot avait déja beaucoup souffert, mais il devait connaitre des heures encore plus sombres et plus angoissantes.

\section{(d suivre)}

André Surprenant, 8.j.

72. Q, 2(1648): 65-66. 